\title{
EVIDENCE FOR TEMPERATURE CHANGE AND OBLIQUE PULSATION FROM LIGHT CURVE FITS OF THE PULSATING WHITE DWARF GD 358
}

\author{
M. H. Montgomery ${ }^{1,2}$, J. L. Provencal ${ }^{2,3}$, A. Kanaan ${ }^{4}$, Anjum S. Mukadam ${ }^{5}$, S. E. Thompson ${ }^{2,3}$, J. Dalessio ${ }^{3}$, \\ H. L. ShipMAn ${ }^{3}$, D. E. Winget ${ }^{1}$, S. O. KePleR ${ }^{6}$, AND D. Koester ${ }^{7}$ \\ ${ }^{1}$ Department of Astronomy and McDonald Observatory, University of Texas at Austin, Austin, TX, USA; mikemon@astro.as.utexas.edu \\ ${ }^{2}$ Delaware Asteroseismic Research Center, Mt. Cuba Observatory, Greenville, DE, USA \\ ${ }^{3}$ Department of Physics and Astronomy, University of Delaware, Newark, DE, USA \\ ${ }^{4}$ Departamento de Física Universidade Federal de Santa Catarina, C.P. 476, 88040-900, Florianópolis, SC, Brazil \\ ${ }^{5}$ Department of Astronomy, University of Washington, Seattle, WA, USA \\ ${ }^{6}$ Instituto de Fíisica UFRGS, C.P. 10501, 91501-970 Porto Alegre, RS, Brazil \\ ${ }^{7}$ Institut für Theoretische Physik und Astrophysik, Universität Kiel, 24098 Kiel, Germany \\ Received 2010 January 7; accepted 2010 April 15; published 2010 May 13
}

\begin{abstract}
Convective driving, the mechanism originally proposed by Brickhill for pulsating white dwarf stars, has gained general acceptance as the generic linear instability mechanism in DAV and DBV white dwarfs. This physical mechanism naturally leads to a nonlinear formulation, reproducing the observed light curves of many pulsating white dwarfs. This numerical model can also provide information on the average depth of a star's convection zone and the inclination angle of its pulsation axis. In this paper, we give two sets of results of nonlinear light curve fits to data on the DBV GD 358. Our first fit is based on data gathered in 2006 by the Whole Earth Telescope; this data set was multiperiodic containing at least 12 individual modes. Our second fit utilizes data obtained in 1996, when GD 358 underwent a dramatic change in excited frequencies accompanied by a rapid increase in fractional amplitude; during this event it was essentially monoperiodic. We argue that GD 358's convection zone was much thinner in 1996 than in 2006, and we interpret this as a result of a short-lived increase in its surface temperature. In addition, we find strong evidence of oblique pulsation using two sets of evenly split triplets in the 2006 data. This marks the first time that oblique pulsation has been identified in a variable white dwarf star.
\end{abstract}

Key words: convection - stars: individual (GD 358) - stars: magnetic field - stars: oscillations - stars: variables: general

Online-only material: color figures

\section{ASTROPHYSICAL CONTEXT}

White dwarf stars offer several advantages for astrophysical study. First, they are the evolutionary endpoint of about $97 \%$ of all stars and are therefore representative of a large fraction of the stellar population. Second, the source of their pressure support is electron degeneracy (Chandrasekhar 1939), so their bulk mechanical structure is well understood. Third, nuclear reactions, if any, contribute a negligible amount to their energy, so their evolution is dominated by simple cooling (Mestel 1952). Finally, they are observed to pulsate in specific temperature ranges. The pulsators are believed to be typical in every other way, so what we learn asteroseismologically about them should apply to all white dwarf stars (for recent reviews, see Winget \& Kepler 2008; Fontaine \& Brassard 2008).

In addition to learning about the stars themselves, the relative simplicity of white dwarfs makes them ideal laboratories for testing and constraining poorly understood physical processes. One such process, convection, is an important energy transfer process in most stars, yet it remains one of the largest sources of uncertainty in stellar modeling. For instance, main-sequence stars at least $20 \%$ more massive than the Sun have convective cores, and the amount of convective overshoot and mixing is the primary factor that determines their main-sequence lifetimes (see, e.g., Di Mauro et al. 2003). In addition, red giants and asymptotic giant branch stars have large convective envelopes, and the details of convection play a role in the evolution of their surface abundances and in their overall evolution (Bertelli et al. 2009).
We have developed a method which uses the pulsations of white dwarf stars to measure fundamental parameters of their convection zones. The physical idea is that the pulsations cause local surface temperature (" $T$ eff") variations that lead to local variations in the depth of the convection zone. As the convection zone waxes and wanes it both absorbs and releases energy, modulating the local energy flux (Brickhill 1991; Goldreich \& Wu 1999). Due to the extreme temperature sensitivity of convection, finite amplitude pulsations can lead to highly nonlinear light curves (Brickhill 1983, 1992; Wu 2001; Ising \& Koester 2001). In Montgomery (2005), we showed how a simple numerical model could be used to obtain not only good light curve fits but also information on the average depth of a star's convection zone and the inclination angle of its pulsation axis.

\section{NONLINEAR LIGHT CURVE FITS}

Montgomery (2005) demonstrated that by considering the nonlinear response of the convection zone (Brickhill 1992; Wu 2001) one could obtain excellent fits to the light curves of two (nearly) single-mode white dwarf pulsators. We have since extended this technique to multiperiodic stars (Montgomery 2007) and have taken into account the nonlinear relationship between the bolometric and observed flux variations (Montgomery 2008). In this section, we describe these effects and show how they have been added to our nonlinear light curve fitting technique.

Our approach is well summarized in Montgomery (2005) and Montgomery (2008). Briefly, we assume that the convective 
turnover time of fluid elements in the convection zone is short $(\lesssim 1 \mathrm{~s})$ compared to the periods of pulsation ( $\gtrsim 100 \mathrm{~s})$, so that the convection zone responds almost instantaneously to the pulsations and is always in hydrostatic equilibrium. In addition, we assume that the perturbation of the flux at the base of the convection zone is sinusoidal. Next, we assume that the luminosity changes are due only to temperature changes and not to geometric effects; Robinson et al. (1982) found that the fractional radius change $\Delta R / R \sim 10^{-5}-10^{-4}$ for the DAVs, with temperature variations (and the associated changes in limb darkening) accounting for well over $99 \%$ of the luminosity variation, and this result was confirmed by Watson (1988) for both the DAV and DBV stars. Finally, the surface convection zones of pulsating white dwarfs are quite thin geometrically, of order $10^{-5}$ of the radius of the star; this allows us to use the planeparallel approximation and neglect horizontal energy transport. With these assumptions, we are able to derive a relationship between the local flux at a given $(\theta, \phi)$ entering the convection zone at its base, $F_{\text {base }}$, and that leaving it at the photosphere, $F_{\text {phot: }}$ :

$$
F_{\text {phot }}=F_{\text {base }}+\tau_{C} \frac{d F_{\text {phot }}}{d t},
$$

where the new timescale $\tau_{C} \equiv \tau_{C}\left(F_{\text {phot }}\right)$ describes the changing heat capacity of the convection zone as a function of the local photospheric flux. We parameterize it as

$$
\tau_{C}=\tau_{0}\left(\frac{T_{\mathrm{eff}}}{T_{\mathrm{eff}, 0}}\right)^{-N}
$$

where $\tau_{0}$ is the equilibrium value of $\tau_{C}, T_{\text {eff }}$ is the instantaneous effective temperature and $T_{\text {eff, } 0}$ is its equilibrium value, and $N$ is a parameter describing the sensitivity of $\tau_{C}$ to changes in $T_{\text {eff }}$. From standard mixing length theory of convection we expect that $N \sim 90$ for DAVs and $N \sim 23$ for DBVs. It is this extreme temperature sensitivity which is responsible for the large nonlinearities seen in white dwarf pulsations. For reference, this timescale is closely related to the standard thermal timescale $\left(\tau_{\mathrm{th}}\right)$ at the base of the convection zone: for the DAVs $\tau_{C} \approx 4 \tau_{\text {th }}$ (Goldreich \& Wu 1999) and for the DBVs $\tau_{C} \approx 0.6 \tau_{\text {th }}$. With the further assumption that the angular dependence of $F_{\text {base }}$ is given by a spherical harmonic $Y_{\ell m}$, we can calculate the bolometric flux changes at the surface of the model and average them appropriately over the visible disk of the model.

\subsection{Improvements in the Modeling}

Since 2005 we have made important technical improvements to the light curve fitting code. First, we extended the code to include the more common multiperiodic case, where many modes with different $\ell$ and $m$ values are simultaneously present. Thus, the flux at the base of the convection zone is now given by a sum over the modes:

$$
\frac{\delta F_{\text {base }}}{F_{\text {base }}}=\operatorname{Re}\left\{\sum_{j=1}^{M} A_{j} e^{i\left(\omega_{j} t+\phi_{j}\right)} Y_{\ell_{j} m_{j}}(\theta, \phi)\right\} .
$$

In this formula, $A_{j}, \omega_{j}, \phi_{j}, \ell_{j}$, and $m_{j}$ are the amplitude, angular frequency, phase, $\ell$, and $m$ values of the $j$ th mode, and the total number of modes is $M$.

Second, we adapted the code to simultaneously fit an arbitrary number of observations ("runs"). This is a necessary step for applying this technique to multiple runs obtained from

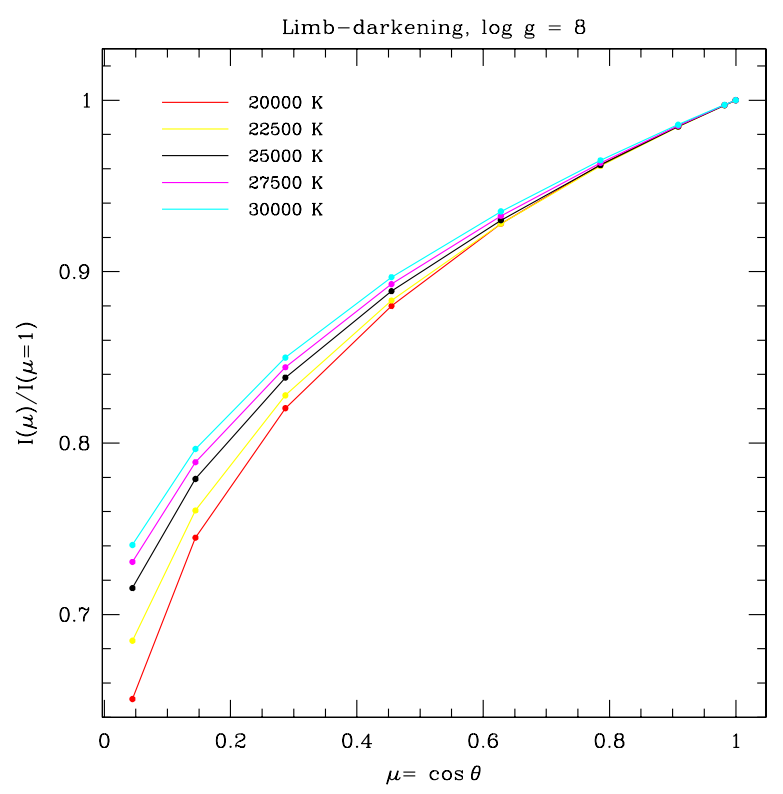

Figure 1. Example of the limb darkening in DB model atmospheres with $\log g=8.0$, for the indicated range of temperatures.

(A color version of this figure is available in the online journal.)

Whole Earth Telescope (WET) campaigns as well as successive nights of single-site observations. Since the code allocates and deallocates memory as needed it typically uses only $8 \mathrm{MB}$ of RAM, independent of the number of runs included in the fit.

Third, we replaced our simple analytical prescription for limb darkening with tabulated values based on a grid of our model atmospheres (for a description of the models, see Koester 2010). This grid ranges in $T_{\text {eff }}$ from $20,000 \mathrm{~K}$ to $30,000 \mathrm{~K}$ in steps of $500 \mathrm{~K}$ and in $\log g$ from 7.5 to 8.5 in steps of 0.25 . From this grid we instantaneously calculate the local flux as a function of $T_{\text {eff }}$ and $\mu \equiv \cos \theta$; thus, variations in the limb darkening with $T_{\text {eff }}$ are automatically included. In Figure 1, we show examples of the limb darkening for a $\log g=8.0 \mathrm{He}$ atmosphere white dwarf model as a function of $\mu$ for a range of $T_{\text {eff }}$ values.

Finally, we improved the way in which the local bolometric flux variations are mapped into variations in a given wavelength band. Previously we used a flux "correction factor," $\alpha_{X}$, to accomplish this. Denoting by $F_{X}$ the flux in the passband $X$, then, for small fractional changes in the fluxes, $\alpha_{X}$ was defined by

$$
\frac{\delta F_{X}}{F_{X}}=\alpha_{X} \frac{\delta F_{\mathrm{phot}}}{F_{\mathrm{phot}}},
$$

where $F_{\text {phot }}$ is the local bolometric flux at the photosphere and $\delta F$ is the variation in the respective fluxes due to the pulsations. Clearly, $\alpha_{X}$ depends on the wavelength coverage of the passband as well as the wavelength distribution of the flux from the source. In previous analyses, we estimated that $\alpha \sim 0.42$ for DBVs and $\alpha \sim 0.66$ for DAVs. The value for the DAVs is not that different from what one obtains from a proper calculation assuming a passband centered on $5000 \AA$. For the DBVs, however, the more detailed calculations yield a value of $\alpha \sim 0.25-0.35$ which is significantly different from the earlier estimates.

Of course, $\alpha$ is not strictly a constant but rather is a function of $T_{\text {eff }}$ and therefore $F_{\text {phot }}$. For larger amplitudes, departures from linearity between the fluxes become more important, and to do the problem properly we need $F_{X}$ as a function of $F_{\text {phot }}$, i.e., $F_{X} \equiv F_{X}\left(F_{\text {phot }}\right)$. 
To calculate this flux conversion we use the same grid of DB model atmospheres described earlier. In each model atmosphere, the flux is tabulated as a function of wavelength $\lambda$ and viewing angle $\mu$, so limb darkening is automatically included. Using a given passband, we integrate it against the model atmosphere flux to obtain $F_{X}$ as a function of $T_{\text {eff }}, \mu$, and $\log g$. Since $\log g$ is essentially constant during the pulsations, we only have to interpolate once between sets of models for the given value of $\log g$; thus, we have $F_{X} \equiv F_{X}\left(T_{\text {eff }}, \mu\right)$. Finally, since $F_{\text {phot }} \propto T_{\text {eff }}^{4}$, we can express this as $F_{X} \equiv F_{X}\left(F_{\text {phot }}, \mu\right)$.

In practice, we are only interested in relative flux changes, so we calculate everything relative to a reference flux, which we take to be the equilibrium flux of the star. If we denote by a subscript " 0 " the equilibrium values of the fluxes, then the function $f$ we want is defined by

$$
\frac{F_{X}}{F_{X, 0}}=f\left(\frac{F_{\text {phot }}}{F_{\text {phot }, 0}}, \mu\right) .
$$

In the top panel of Figure 2, we show the fluxes $F_{X} / F_{X, 0}$ and $F_{\text {phot }} / F_{\text {phot, } 0}$ as a function of $T_{\text {eff }}$, both normalized to one at $T_{\text {eff }}=25,000 \mathrm{~K}$; the filter $X$ is assumed to have an effective wavelength of $\sim 5200 \AA$. The response of this filter was obtained from convolving the wavelength response of the ALFOSCFASU CCD, the Nordic Telescope (NOT) primary and secondary mirror reflectivities, an S8612 filter, and atmospheric absorption. In the lower panel, we show the function $f$ defined in Equation (5) as derived from these calculations. We see that while this is not a perfectly linear relationship, the deviations from linearity are not dramatic. Thus, while we use the fully nonlinear relation in our calculations, we expect this effect to make only a minor contribution to the overall nonlinearities associated with the pulsations.

\section{LIGHT CURVE FITS TO THE 2006 WET RUN}

Our recent work with light curve fitting has been limited to nearly single-mode pulsators: the DAVs G29-38 ${ }^{8}$ and GD 154 , and the DBV PG1351+489. This is because (1) monoperiodic data can be folded at the pulsation period, producing a high signal-to-noise ratio $(\mathrm{S} / \mathrm{N})$ "light curve" and (2) the number of possible mode identifications ( $\ell$ and $m$ values) for a single mode is small enough that all possibilities can be directly explored.

GD 358 violates both of these conditions. First, due to the nonlinear interaction of its large amplitude modes, the pulse shape obtained by folding its light curve at a mode period is not the same as the pulse shape which would be obtained in the absence of other modes (Montgomery 2007). Second, GD 358 has a large number of observed modes (see Table 2), and it is impractical to search all possible combinations of $\ell$ and $m$ values which each mode can take. For instance, if we assume GD 358 to have of order $\sim 10$ modes, all of which have $\ell=1$, then all possible permutations of $m$ values yield a number of $(2 \ell+1)^{10} \sim 60,000$ different cases. Since each fit takes of order an hour on a single processor computer, this is completely impractical using a standard desktop computing approach.

Fortunately, GD 358 is well studied, so we have a good idea what the $\ell$ and $m$ values for the main pulsation modes are (e.g., Metcalfe et al. 2000; Winget et al. 1994). Even so, our derived values of $\tau_{0}$ depend only weakly on the assumed mode

\footnotetext{
8 More precisely, while G29-38 is normally multiperiodic, the data set used by Montgomery (2005) was taken at a time when its light curve was dominated by a single large mode.
}
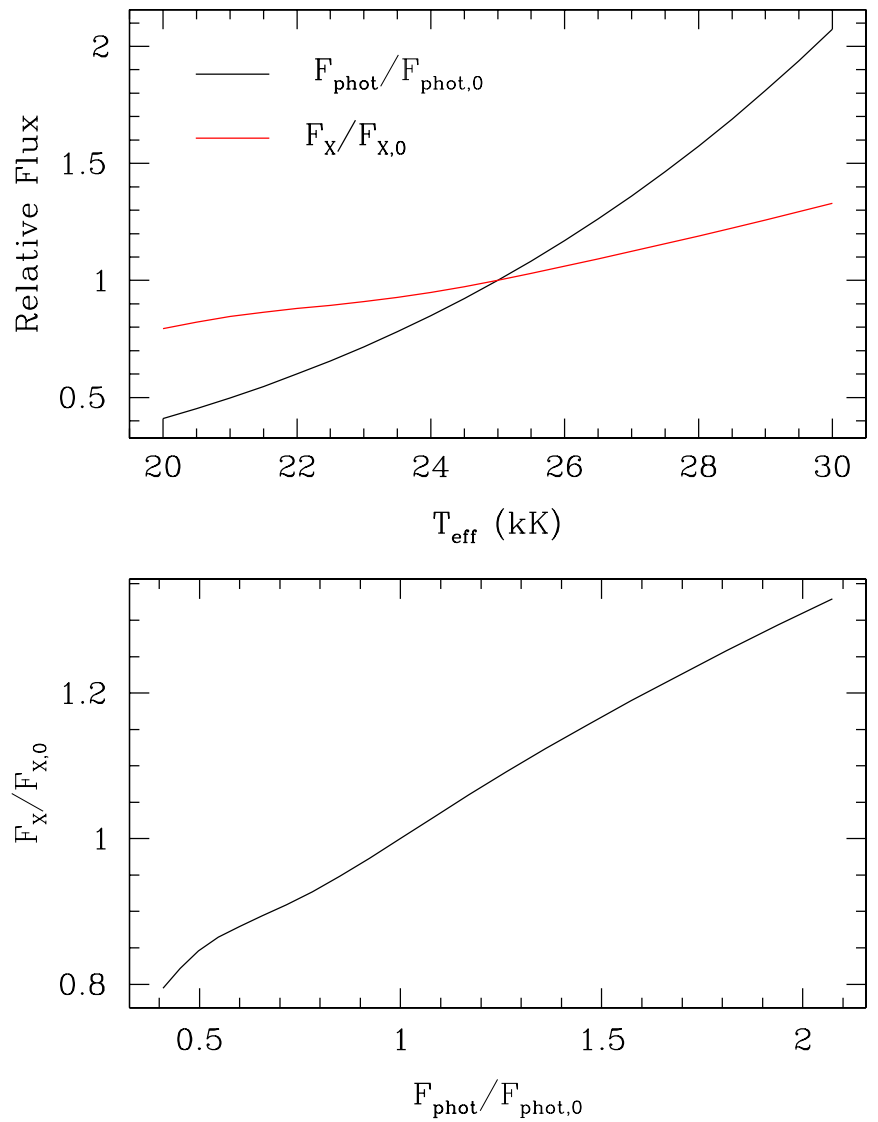

Figure 2. Top panel: the bolometric flux $\left(F_{\text {phot }}\right)$ and the flux in the given passband $\left(F_{X}\right)$ as a function of $T_{\text {eff }}$ for $\mu=1$. These calculations are for a $\log g=8.0 \mathrm{DB}$ model, and both fluxes have been normalized to one at $T_{\text {eff }}=25,000 \mathrm{~K}$. Lower panel: the passband flux as a function of the bolometric flux, again normalized at $T_{\text {eff }}=25,000 \mathrm{~K}$. This is the function $f$ given by Equation (5).

(A color version of this figure is available in the online journal.)

identifications, so it is not necessary for the mode identification of each mode to be exact. Furthermore, the extended time baseline and excellent coverage of the 2006 WET run allow us to obtain very accurate frequencies for these modes (Provencal et al. 2009). With this as a basis, our approach is to assume that the frequencies and mode identifications are known and then to make nonlinear light curve fits to a subset of runs in the WET campaign which have high $\mathrm{S} / \mathrm{N}$. This implicitly assumes that the pulsations are coherent throughout the time spanned by the runs; in Section 6, we show that this is not strictly true for some of the modes. High $\mathrm{S} / \mathrm{N}$ data are desirable since we are mainly interested in the nonlinear part of the light curve, which itself is smaller than the linear part.

Many high quality runs were taken during the 2008 May WET run, and we list in Table 1 those used in our fits. We included the 12 largest amplitude periodicities from the 2006 WET run which were deemed to be independent frequencies and not linear combinations, and these are given in Table 2. The $\ell$ and $m$ identifications are taken from previous analyses of this star (Winget et al. 1994; Kepler et al. 2003; Metcalfe 2003; Provencal et al. 2009) and are based on asymptotic theory as well as genetic algorithm fits. To calculate the conversion from bolometric to observed passband fluxes, we must assume values for $T_{\text {eff }}$ and $\log g$. We chose the values of Beauchamp et al. (1999), $T_{\text {eff }}=24,900 \mathrm{~K}$ and $\log g=7.91$, although we have also used those of Castanheira et al. $\left(2005 ; T_{\text {eff }}=24,100 \mathrm{~K}\right.$, $\log g=7.91$ ) to assess the uncertainties this choice introduces. 
Table 1

Observing Runs Used for Light Curve Fits

\begin{tabular}{|c|c|c|c|}
\hline Run Name & Telescope & Instrument & Length (hr) \\
\hline chin 20060527 & BAO $2.16 \mathrm{~m}$ & PMT & 4.5 \\
\hline $\operatorname{chin} 20060528$ & BAO $2.16 \mathrm{~m}$ & PMT & 5.2 \\
\hline chin 20060531 & BAO $2.16 \mathrm{~m}$ & PMT & 3.9 \\
\hline hawa20060518 & $0.6 \mathrm{~m}$ & Apogee & 2.2 \\
\hline hawa20060519 & $0.6 \mathrm{~m}$ & Apogee & 1.7 \\
\hline hawa20060520 & $0.6 \mathrm{~m}$ & Apogee & 3.5 \\
\hline hawa20060521 & $0.6 \mathrm{~m}$ & Apogee & 7.8 \\
\hline hawa20060522 & $0.6 \mathrm{~m}$ & Apogee & 6.6 \\
\hline hawa20060523 & $0.6 \mathrm{~m}$ & Apogee & 3.7 \\
\hline hawa20060524 & $0.6 \mathrm{~m}$ & Apogee & 5.7 \\
\hline hawa20060525 & $0.6 \mathrm{~m}$ & Apogee & 8.8 \\
\hline hawa20060526 & $0.6 \mathrm{~m}$ & Apogee & 9.1 \\
\hline hawa20060527 & $0.6 \mathrm{~m}$ & Apogee & 7.8 \\
\hline hawa20060528 & $0.6 \mathrm{~m}$ & Apogee & 9.1 \\
\hline hawa20060530 & $0.6 \mathrm{~m}$ & Apogee & 8.7 \\
\hline kpno20060518 & KPNO $2.1 \mathrm{~m}$ & Apogee & 7.0 \\
\hline kpno20060519 & KPNO $2.1 \mathrm{~m}$ & Apogee & 7.3 \\
\hline kpno20060520 & KPNO $2.1 \mathrm{~m}$ & Apogee & 7.6 \\
\hline kpno20060521 & KPNO $2.1 \mathrm{~m}$ & Apogee & 7.3 \\
\hline kpno20060522 & KPNO $2.1 \mathrm{~m}$ & Apogee & 1.0 \\
\hline kpno20060523 & KPNO $2.1 \mathrm{~m}$ & Apogee & 2.9 \\
\hline mcdo20060523 & $2.1 \mathrm{~m}$ & Argos & 7.4 \\
\hline mcdo20060524 & $2.1 \mathrm{~m}$ & Argos & 7.2 \\
\hline mcdo20060525 & $2.1 \mathrm{~m}$ & Argos & 6.4 \\
\hline mcdo20060528b & $2.1 \mathrm{~m}$ & Argos & 7.2 \\
\hline medo20060529 & $2.1 \mathrm{~m}$ & Argos & 8.2 \\
\hline nord20060607 & $2.7 \mathrm{~m}$ & ALFOSC & 7.1 \\
\hline nord 20060608 & $2.7 \mathrm{~m}$ & ALFOSC & 8.0 \\
\hline nord20060609 & $2.7 \mathrm{~m}$ & ALFOSC & 7.9 \\
\hline
\end{tabular}

Table 2

Independent Modes from the 2006 WET Run

\begin{tabular}{crrr}
\hline $\begin{array}{c}\text { Period } \\
(\mathrm{s})\end{array}$ & $k$ & $\ell$ & $m$ \\
\hline 422.56 & 8 & 1 & 1 \\
423.90 & 8 & 1 & -1 \\
463.38 & 9 & 1 & 1 \\
464.21 & 9 & 1 & 0 \\
465.03 & 9 & 1 & -1 \\
571.74 & 12 & 1 & 1 \\
574.16 & 12 & 1 & 0 \\
575.93 & 12 & 1 & -1 \\
699.68 & 15 & 1 & 0 \\
810.29 & 18 & 1 & 0 \\
852.50 & 19 & 1 & 0 \\
962.38 & 22 & 1 & 0 \\
\hline
\end{tabular}

We began the fitting process with the highest $\mathrm{S} / \mathrm{N}$ data taken with the $2.7 \mathrm{~m}$ NOT. However, it became apparent that GD 358's complex pulsation spectrum required an extended baseline of data to constrain the phases of closely spaced frequencies. Fortunately, GD 358's brightness and large amplitude meant that a large number of individual observing runs met the required $\mathrm{S} / \mathrm{N}$.

Our simultaneous fit to the runs in Table 1 yields the following parameters: $\tau_{0}=572.9 \pm 6.1 \mathrm{~s}, N=23.5 \pm 0.1$, and $\theta_{i}=$ $50.5 \pm 0.2$. Figure 3 shows the ability of the fit to reproduce the essential features of the light curve (run mcdo20060523 plotted). Additional results for this fit, including the amplitude and phase for each mode, are given in Table 3 . The given error bars are formal and should be treated as lower bounds.
Table 3

Simultaneous Fit to GD 358 Data Set: $\tau_{0}=572.9 \pm 6.1 \mathrm{~s}, N=23.5 \pm 0.1$, $\theta=50.5 \pm 0.2$

\begin{tabular}{llrcc}
\hline \hline Period & $\ell$ & $m$ & Amplitude & Phase (rad) \\
\hline 962.385 & 1 & 0 & $0.1087 \pm 0.0012$ & $2.4641 \pm 0.0069$ \\
852.502 & 1 & 0 & $0.1198 \pm 0.0015$ & $3.3007 \pm 0.0075$ \\
810.291 & 1 & 0 & $0.4581 \pm 0.0049$ & $3.1301 \pm 0.0030$ \\
575.933 & 1 & -1 & $0.4838 \pm 0.0051$ & $3.4434 \pm 0.0039$ \\
574.162 & 1 & 0 & $0.2257 \pm 0.0024$ & $5.5258 \pm 0.0062$ \\
573.485 & 1 & 0 & $0.1082 \pm 0.0016$ & $3.6576 \pm 0.0115$ \\
571.735 & 1 & 1 & $0.3728 \pm 0.0040$ & $2.4489 \pm 0.0046$ \\
465.034 & 1 & -1 & $0.1408 \pm 0.0021$ & $1.8310 \pm 0.0123$ \\
464.209 & 1 & 0 & $0.1391 \pm 0.0018$ & $2.4672 \pm 0.0097$ \\
463.376 & 1 & 1 & $0.2540 \pm 0.0030$ & $0.2052 \pm 0.0073$ \\
423.898 & 1 & -1 & $0.2406 \pm 0.0030$ & $2.0455 \pm 0.0083$ \\
422.561 & 1 & 1 & $0.2537 \pm 0.0031$ & $0.6020 \pm 0.0077$
\end{tabular}
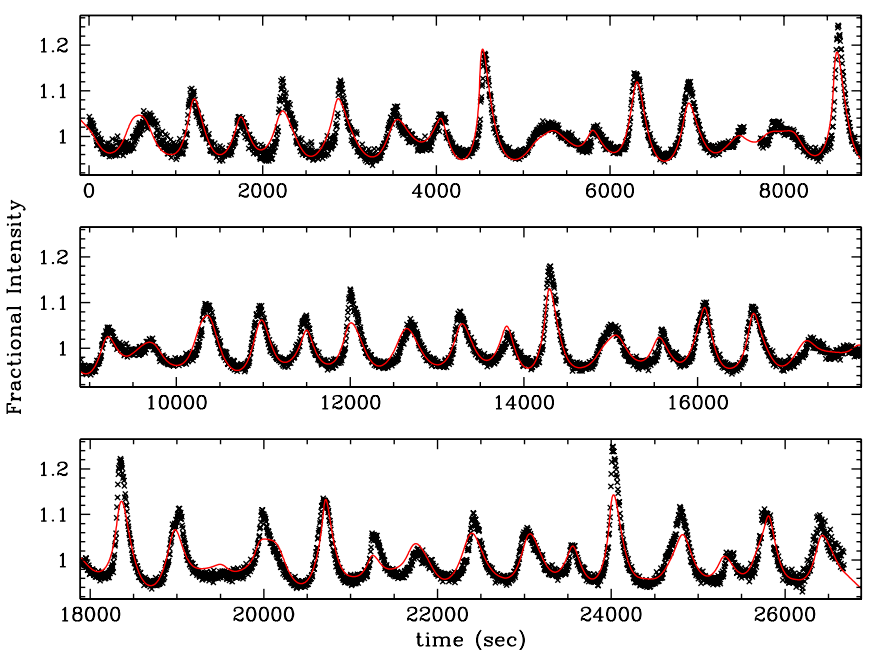

Figure 3. Comparison of the simultaneous fit of Table 3 (solid line) to the light curve from run mcdo20060523 (crosses).

(A color version of this figure is available in the online journal.)

To test the sensitivity of the value of $\tau_{0}$ to the $\ell$ identifications, we re-computed fits changing the assumed $\ell$ of the largest amplitude mode $(P=810.29 \mathrm{~s})$ from $\ell=1$ to $\ell=2$. With this assumption, the best fit resulted for an identification with $\ell=2, m=1$ and had the following parameter values: $\tau_{0}=569.5 \pm 7.6 \mathrm{~s}, N=14.5 \pm 0.1$, and $\theta_{i}=61.3 \pm 0.1$. This indicates that the value obtained for $\tau_{0}$ does not crucially depend on the mode identifications of each mode in the fit.

Examination of the fit for each individual run revealed an apparent modulation of amplitudes, i.e., on some nights the variations in the light curve were smaller than the fit and on other nights they were larger. In addition, looking at data from single sites suggested that the change from a smaller to a larger amplitude state alternated on a roughly night-to-night timescale.

To test whether a geometric effect or a change in the background state of the star could be causing the night-to-night modulation of the amplitudes, we went back and re-fit each run in Table 1 individually. For these fits we fixed the period and amplitude of each mode to be those given in Table 3, but we allowed the inclination angle $\theta_{i}$ and the phases of the modes to vary. We also allowed $\tau_{0}$ and $N$ to vary. Thus, from each run we obtained a best-fit value of the parameters at the time of the run. In Figure 4, we present the results of this procedure; we plot the variations in $\theta_{i}, N$, and $\tau_{0}$ as a function of time. While no clear trends are seen in $\tau_{0}$ and $N$ (lower panels), the 


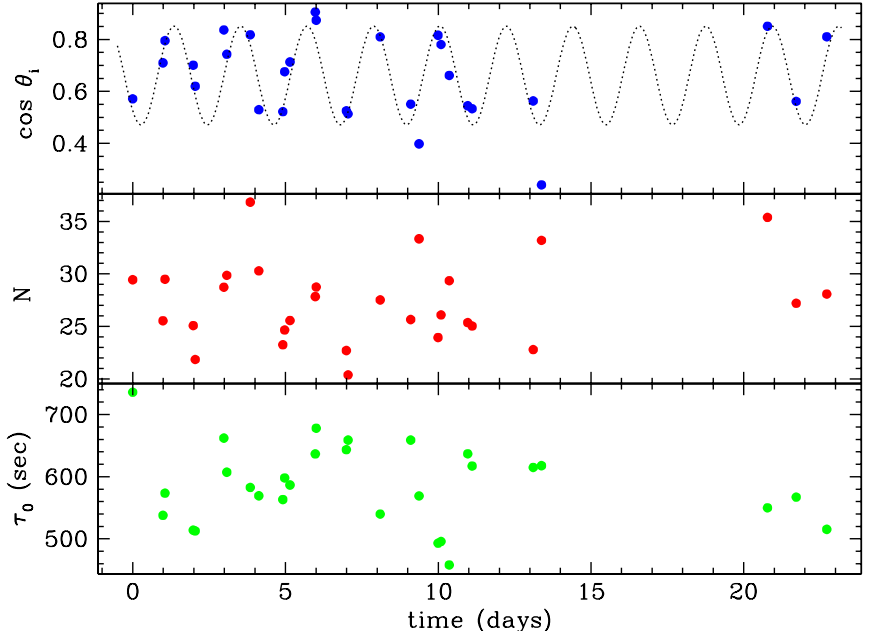

Figure 4. Variation of $\theta_{i}, N$, and $\tau_{0}$ as a function of time during the 2006 WET run. While $\tau_{0}$ and $N$ show no obvious signatures, $\theta_{i}$ shows variations which may be sinusoidal in origin.

(A color version of this figure is available in the online journal.)

variations in $\theta_{i}$ are suggestive of a periodic origin (top panel). Assuming a sinusoidal variation, we have included the bestfit sine curve in this plot; it has an amplitude of $14.9 \pm 1.8$ and a period of $2.17 \pm 0.01$ days. On the other hand, if we ignore the possible origin of these variations and simply consider them to be separate measures of $\tau_{0}, N$, and $\theta_{i}$, then we obtain fairly conservative limits on the error of the mean values of these quantities: $\tau_{0}=586.0 \pm 11.7 \mathrm{~s}, N=27.4 \pm 0.7$, and $\theta_{i}=47.5 \pm 2.2$. We return to the question of this modulation in Section 6.

\section{THE SFORZANDO}

During the 2006 WET run, GD 358 was stable in that the amplitudes and phases of its modes were fairly constant over the 3 week length of the run (except for the modulation detailed above and in Section 6). However, GD 358 is known to change its pulsation spectrum on a range of timescales. A spectacular example of this behavior occurred in 1996. Within a period of $36 \mathrm{hr}$, all of the power in the high- $k$ range (pulsations with periods $\gtrsim 700 \mathrm{~s}$ ) disappeared within detection limits. At the same time, GD 358 more than doubled its apparent pulsation amplitude, with power appearing almost exclusively at lower $k$, with a period of $\sim 420 \mathrm{~s}$. Over the next week, its amplitude decreased to "normal" levels, while the high- $k$ power did not return for approximately 1 month. This dramatic change is documented in several papers, most recently in Kepler et al. (2003) and Provencal et al. (2009). The episode itself is termed the "sforzando" after a musical term for a sudden and short-lived increase in loudness.

In Figure 5, we show a section of GD 358's light curve during the sforzando. Ironically, during this dramatic episode the light curve was much simpler. While the amplitude was larger, the star was essentially a single-mode pulsator, and the individual pulses were more sinusoidal (less nonlinear) than before. Still, GD 358 was in a transient state and not all pulses were identical. The upper and lower panels of Figure 5 show pulses significantly below and above the fit, while the middle panel seems to represent a repeating, "quasi-static" state. Even with these caveats, the relative simplicity of these data is ideal for nonlinear light curve fitting.

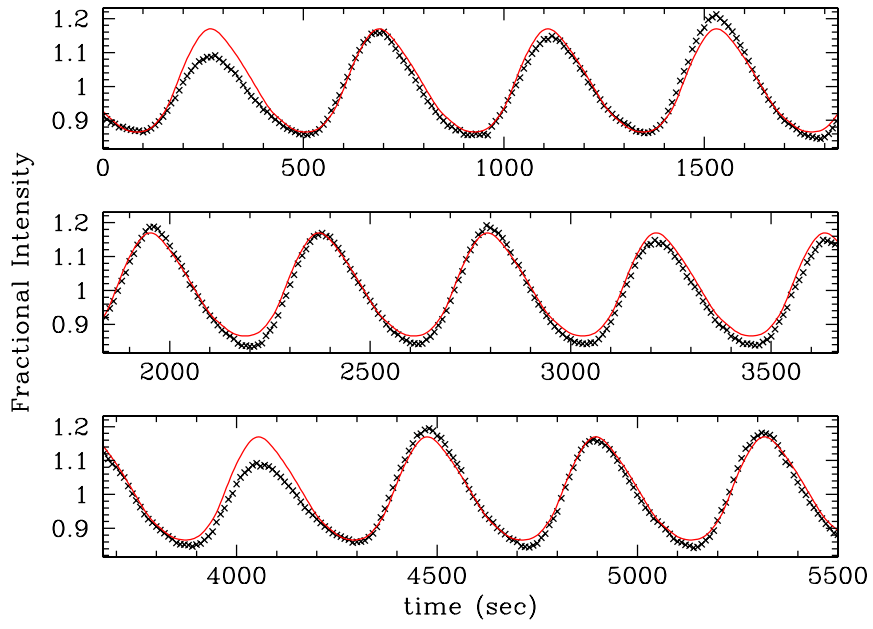

Figure 5. Section of the light curve of GD 358 during the sforzando event in 1996. The crosses are the data points and the curve is the best nonlinear light curve fit.

(A color version of this figure is available in the online journal.)

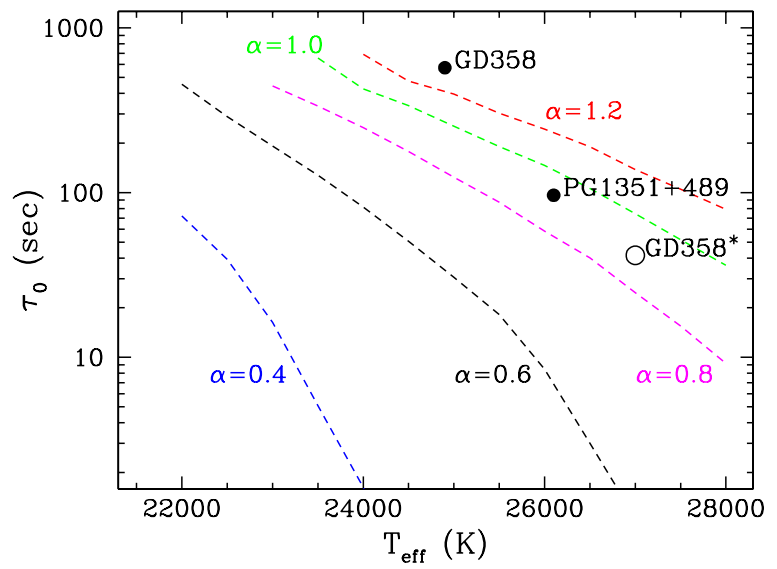

Figure 6. Comparison of the derived convective parameters $\tau_{0}$ with values expected from ML2/ $\alpha$ convection. The labeled points are individual objects and the dashed curves are the calculations. The label "GD 358" represents GD 358 during the 2006 WET run while "GD 358*" stands for GD 358 during its sforzando episode.

(A color version of this figure is available in the online journal.)

From a time series analysis of this short section of data, we find that these pulses have a period of approximately $420.7 \mathrm{~s}$. Assuming $\ell=1$ and trying all values of $m$, we find the best fit shown in Figure 5: $m=0, \tau_{0}=41.6 \pm 2.3 \mathrm{~s}, N=3.6 \pm 0.2$, and $\theta_{i}=56.1 \pm 1.1$. For $\ell=1, m=1$ a similar quality fit can be found, but it has a less plausible inclination angle $\left(\sim 85^{\circ}\right)$ that is not consistent with the values of $\theta_{i}$ previously found in this paper; even so, $\tau_{0}=28.1 \mathrm{~s}$ for this fit. If we assume $\ell=2$ then the best fit has $\tau_{0} \sim 24.2 \mathrm{~s}$ and $m=0$, although it requires such a large intrinsic amplitude that we consider it unphysical. Summing up, while we have good reasons for preferring the $\ell=1, m=0$ identification for our fits, the overall value of $\tau_{0}$ is not strongly dependent on this identification.

Comparing these results to those of Section 3, we find that $\tau_{0}$ was much smaller during the sforzando than it was during the 2006 WET run. This implies that, for whatever reason, GD 358's convection zone was thinner during the sforzando. As we can see from Figure 6, this would imply a $T_{\text {eff }}$ several thousand degrees hotter than its normal temperature. For ML2/ $\alpha=1.0$ convection (e.g., Böhm \& Cassinelli 1971), this 


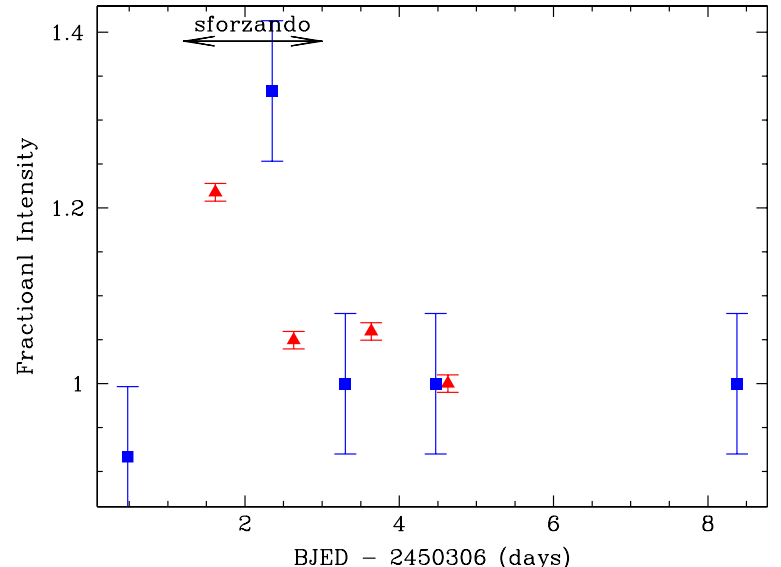

Figure 7. Relative intensity of GD 358 as measured relative to its levels after the sforzando episode. The triangles are data from McDonald Observatory and the squares are data from Mt. Suhora Observatory. Both data sets indicate a jump in intensity near BJED 2450311.

(A color version of this figure is available in the online journal.)

would mean $T_{\text {eff }} \sim 27,000 \mathrm{~K}$, which is two to three thousand degrees hotter than its normal equilibrium state.

Such an increase in temperature would lead to about a $40 \%$ increase in the bolometric luminosity. From Figure 2, we see that such an increase in the bolometric luminosity translates into a $15 \%-20 \%$ increase in intensity as measured in a passband centered at $5200 \AA$. While such a passband is reasonable for a $\mathrm{CCD}+\mathrm{BG} 40 / \mathrm{S} 8612$ filter + atmosphere, the observations in 1996 were made with phototubes, and these are much more blue sensitive. Thus, for a phototube passband centered around $3800 \AA$ A we expect intensity increases of about $20 \%-25 \%$.

As reported in Table 5 of Provencal et al. (2009), such an increase in intensity was observed in GD 358 during the sforzando event. In Figure 7, we plot these data, where the triangles are data from McDonald Observatory and the squares are data from Mt. Suhora Observatory in Poland. Both curves are normalized to a value of 1 near BJED 2450311. We see that these data, although sparse, strongly suggest that GD 358 underwent a sharp intensity increase and decline of about 20\%-30\% during this event. This corroborates our earlier interpretation that GD 358 had a thinner convection zone with a smaller value of $\tau_{0}$, and that this thinness was due to its surface layers being temporarily hotter.

We note that similar results and conclusions have previously been made by Weidner \& Koester (2003). Using the approach of Ising \& Koester (2001) they were the first to make numerical simulations of GD 358's light curve during the sforzando event. As in our present analysis, they found that the shape of the light curve could only be reproduced for higher effective temperatures than normally assumed for this star, suggesting a value of $T_{\text {eff }} \sim 27,000 \mathrm{~K}$.

\subsection{The Amplitude of the $k=8$ Mode}

The physical origin of the sforzando event is completely unknown. All we know for certain is that the amplitudes of the high- $k$ modes rapidly decreased, leaving power in the $k=8$ mode, which itself increased in amplitude by approximately a factor of 30. While we have no model to explain the disappearance of power in the high- $k$ modes, in this section we consider what would happen to the apparent amplitude of the $k=8$ mode if the convection zone were suddenly removed.
One effect of the thermal response of the convection zone is to attenuate the amplitude of the flux variations incident on its base. It acts as a low-pass filter, reducing the photospheric amplitude of a mode according to

$$
\frac{\Delta F_{\text {phot }}}{F_{0}}=\frac{1}{\sqrt{1+\left(\omega \tau_{0}\right)^{2}}} \frac{\Delta F_{\text {base }}}{F_{0}},
$$

where $F_{0}$ is the equilibrium value of the flux, $F_{\text {base }}$ and $F_{\text {phot }}$ are the instantaneous fluxes at the base of the convection zone and at the photosphere, respectively, and $\omega$ is the angular frequency of the mode (Goldreich \& Wu 1999; Wu \& Goldreich 1999).

If we assume that the amplitude of the $k=8$ mode at the base of the convection zone was constant throughout the sforzando, Equation (6) shows that a decrease in $\tau_{0}$ would naturally lead to an increase in observed amplitude. Thus, for a $420 \mathrm{~s}$ mode we would see an increase of approximately a factor of 8 in its apparent amplitude as $\tau_{0}$ goes from $\sim 600 \mathrm{~s}$ to $\sim 40 \mathrm{~s}$. This is a large factor, but it is still much less than the factor of $\sim 30$ increase that was actually observed. Thus, the $k=8$ appears to have increased its intrinsic amplitude by of order a factor of 4 during the sforzando. How it was able to do this on so short a timescale remains a mystery.

\section{THE CONVECTIVE TIME-SCALE $\tau_{0}$}

In Figure 6, we plot the known determinations of the convective response timescale, $\tau_{0}$, versus $T_{\text {eff }}$ for the DBVs. Based on the discussion in the previous section, GD 358 is plotted twice: once for the 2006 data and once for the 1996 data. In addition, we show the position of the DBV PG1351+489 $\left(\tau_{0} \sim 100 \mathrm{~s}\right.$; Montgomery 2005), where we have assumed the pure He fit for its $T_{\text {eff }}$ (Beauchamp et al. 1999). As expected, the data indicate an increase in the depth/mass of the convection zone with decreasing $T_{\text {eff }}$.

We also plot lines in Figure 6 showing the predictions of ML2/ $\alpha$ convection (Böhm \& Cassinelli 1971) for various values of $\alpha$. We see that while low values of $\alpha$ are excluded $(\alpha \lesssim 0.6)$, values in the range 1.0-1.2 provide a reasonable description of how $\tau_{0}$ varies with $T_{\text {eff }}$. We note that the $\log g$ values determined for these stars are nearly identical, so they $d o$ form an actual sequence in $T_{\text {eff }}$. In general, however, $\tau_{0}$ is also a function of $\log g$, albeit a somewhat weaker one.

Our ultimate goal is to map $\tau_{0}$ as a function of both $T_{\text {eff }}$ and $\log g$ for both the DBV and DAV instability strips. This will provide important reference points for new hydrodynamic simulations of convection which are starting to come online (e.g., Muthsam et al. 2010). For instance, for a given white dwarf one can use the measured $T_{\text {eff }}$ and $\log g$ values and perform a hydrodynamic simulation of its convection zone. Then, using Equations (3) and (5) of $\mathrm{Wu}$ (2001) one can compute $\tau_{0}$. This value of $\tau_{0}$ can then be compared with the value derived from the light curve fits outlined above.

\section{THE OBLIQUE PULSATOR MODEL}

\subsection{The Formalism}

In Section 3, we found evidence of a modulation of the inclination angle $\theta_{i}$ (see Figure 4). This modulation has a formal significance level of 14.9/1.8 $\sim 8 \sigma$, which cries out for a physical interpretation. The most obvious is some form of the "oblique pulsator" model in which the pulsation axis is inclined with respect to the rotation axis, and this axis precesses as the 
Table 4

Frequency Solution for Oblique Pulsation Model: $f_{\text {rot }}=5.362 \pm 0.003 \mu \mathrm{Hz}$

\begin{tabular}{lrccc}
\hline \hline Frequency $(\mu \mathrm{Hz})$ & Amplitude $(\mathrm{mma})$ & Phase $(\mathrm{rad})$ & $\Delta \Phi / 2 \pi$ & Independent Frequencies $^{\mathrm{a}}$ \\
\hline \multicolumn{5}{c}{ Triplet 1} \\
\hline $1736.302 \pm 0.004$ & $16.77 \pm 0.13$ & $0.739 \pm 0.008$ & \\
$1741.664 \pm 0.003$ & $10.81 \pm 0.13$ & $2.832 \pm 0.012$ & $0.510 \pm 0.013$ & $1736.302 \pm 0.001$ \\
$1747.027 \pm 0.004$ & $1.75 \pm 0.13$ & $1.724 \pm 0.075$ & & $1741.665 \pm 0.001$ \\
\hline \multicolumn{5}{c}{ Triplet 2 } \\
\hline $1738.362 \pm 0.005$ & $0.95 \pm 0.13$ & $1.268 \pm 0.138$ & \\
$1743.725 \pm 0.003$ & $5.56 \pm 0.13$ & $0.174 \pm 0.023$ & $0.517 \pm 0.023$ & $1737.962 \pm 0.007$ \\
$1749.087 \pm 0.005$ & $12.55 \pm 0.13$ & $2.117 \pm 0.011$ & & $1743.738 \pm 0.002$ \\
& & &
\end{tabular}

Note. ${ }^{\text {a }}$ These are the unconstrained frequency fits of Provencal et al. (2009).

star rotates (Kurtz \& Shibahashi 1986; Kurtz 1982). A magnetic field that is inclined to the rotation axis is usually invoked, and the pulsations are assumed to be aligned with the magnetic axis.

While this hypothesis does introduce several unknowns (a magnetic field, the angle between the magnetic and rotation axes, etc.) it also makes three testable predictions. If we take $f$ and $f_{\text {rot }}$ to be the mode frequency in the frame of the star and the rotational frequency, respectively, then an $\ell=1$ mode with frequency $f$ aligned with the magnetic axis will appear as three separate peaks in the Fourier transform (FT) of the light curve, with frequencies of $f-f_{\text {rot }}, f$, and $f+f_{\text {rot }}$. For clarity, we will refer to these peaks as "geometric" peaks as they only appear because the pulsation axis spins around the rotation axis, leading to a periodic apparent amplitude modulation of the mode. This amplitude modulation manifests itself in the FT as two additional "geometric" peaks on either side of the original frequency, with the beating of these three peaks producing the periodic amplitude changes. As this splitting is caused by the rotation of the star, these frequencies must be equally split to within measurement errors. This is the first and most stringent condition the model must face.

Second, the oblique pulsator model predicts a specific phase relationship for the peaks in a given geometric triplet (or for higher $\ell$, a $(2 \ell+1)$-multiplet). From Kurtz \& Shibahashi (1986), an $\ell=1$ mode generically has luminosity variations given by

$$
\begin{aligned}
\Delta L / L= & A_{-} \cos [(\omega-\Omega) t+\phi] \\
& +A_{0} \cos [\omega t+\phi]+A_{+} \cos [(\omega+\Omega) t+\phi],
\end{aligned}
$$

where $\omega$ and $\Omega$ are $2 \pi$ times $f$ and $f_{\text {rot }}$, respectively (see the Appendix for the complete expressions). These components will only have the same phase $\phi$ for a particular choice of the zero point of time. For other zero points one can show that this phase relation translates to

$$
2 \Phi_{0}-\left(\Phi_{+}+\Phi_{-}\right)=0,
$$

where $\left\{\Phi_{-}, \Phi_{0}, \Phi_{+}\right\}$are the measured phases of the respective components. In general, the product $A_{-} \times A_{+}$can be negative. For this case, if we define the amplitudes always to be positive and absorb any minus signs into the phase for each peak, the relation becomes

$$
2 \Phi_{0}-\left(\Phi_{+}+\Phi_{-}\right)=\pi
$$

Defining $\Delta \Phi \equiv 2 \Phi_{0}-\left(\Phi_{+}+\Phi_{-}\right)$, then from the sign of the amplitudes in Equations (A2)-(A4) we see that $\Delta \Phi / 2 \pi=0$ for $m=0$ modes and $\Delta \Phi / 2 \pi=0.5$ for $m= \pm 1$ modes. These phase relations, while less iron-clad than the equal spacing of the triplets, should be satisfied within the errors for geometric peaks split by oblique rotation.

A third condition/prediction is the relative amplitudes of the geometric peaks. Such a calculation makes assumptions concerning the nature and strength of the magnetic field, so this prediction of the model is the least reliable of the three. In Equations (A2)-(A4) in the Appendix, we give expressions for the amplitudes of the various components of $\ell=1$ modes perturbed by a magnetic field and oblique rotation. The relevant parameters are the inclination angle of the rotation axis, $\theta_{i}$, the obliquity of the magnetic axis, $\beta$, and $x_{1}$. The parameter $x_{1}$ is given by

$$
x_{1} \equiv \frac{\omega_{0}^{(1)}-\omega_{1}^{(1)}}{C_{k \ell} \Omega},
$$

where $C_{k \ell}$ is the rotational splitting coefficient due to the Coriolis force, $\Omega$ is the angular frequency of rotation, and $\omega_{0}^{(1)}$ and $\omega_{1}^{(1)}$ are the perturbations to the frequencies of the $m=0$ and $m=1$ intrinsic modes due to the magnetic field, respectively. Even though this is clearly a more model dependent statement, we would still hope that the amplitudes could approximately be fit and/or predicted within the oblique pulsator formalism.

\subsection{The Results}

First, we consider a fit to the $k=12$ region of the FT, from 1730 to $1750 \mu \mathrm{Hz}$. Provencal et al. (2009) found six significant peaks in this region. We interpret these peaks as resulting from two components of an $\ell=1$ mode, each split into a geometric triplet by oblique pulsation. These two original peaks are part of an intrinsic triplet produced by standard rotational splitting, but the amplitude of the third member is below our detection threshold.

We fit two sets of exactly evenly split triplets to the data set, where the central frequencies of each triplet are 1741.664 and $1743.725 \mu \mathrm{Hz}$, and the value of the splitting is $5.362 \mu \mathrm{Hz}$. The values of the fit parameters we obtained are given in Table 4. In Figure 8, we show the result of pre-whitening the $k=12$ region by this solution. The reduction in power of the FT is very significant, showing that evenly split triplets are a good representation of the data. This is a necessary condition for the oblique pulsator model to be applicable.

Second, we consider the phase relations within each of these equally split triplets. From Table 4, we see that the first triplet has $\Delta \Phi / 2 \pi=0.510 \pm 0.013$ and that the second triplet has $\Delta \Phi / 2 \pi=0.517 \pm 0.023$. The oblique pulsator model also passes this test with flying colors. In addition, the fact that $\Delta \Phi / 2 \pi \sim 0.5$ rather than 0.0 implies that the modes are not axisymmetric, i.e., $|m|=1$ for both modes. For whatever 


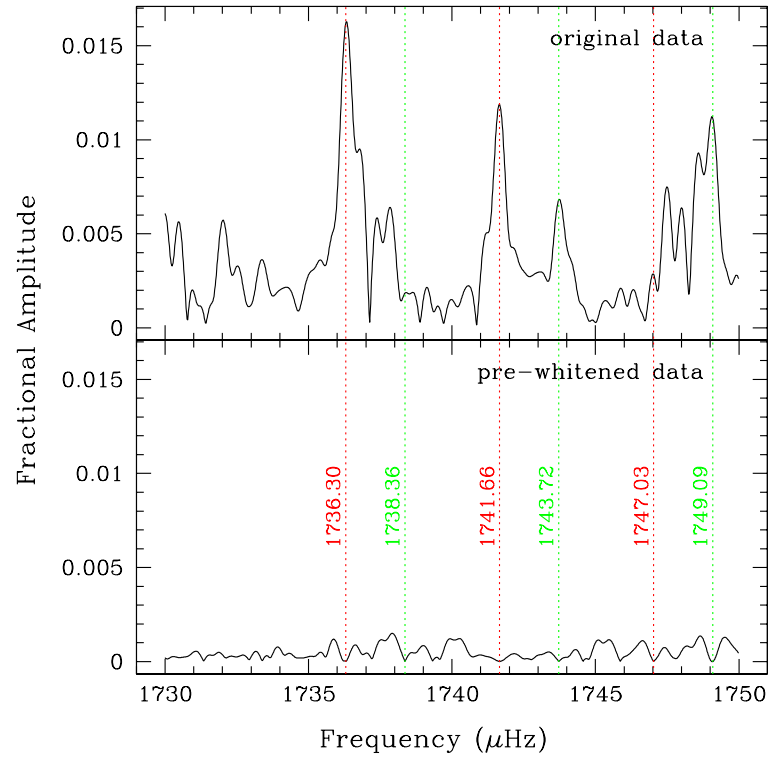

Figure 8. Effect of pre-whitening by two identically split triplets in the oblique pulsator model. The top panel is the original FT of the $k=12$ region and the lower panel is the result of pre-whitening by the two pairs of triplets. The splitting within each triplet is $5.362 \pm 0.003 \mu \mathrm{Hz}$.

(A color version of this figure is available in the online journal.)

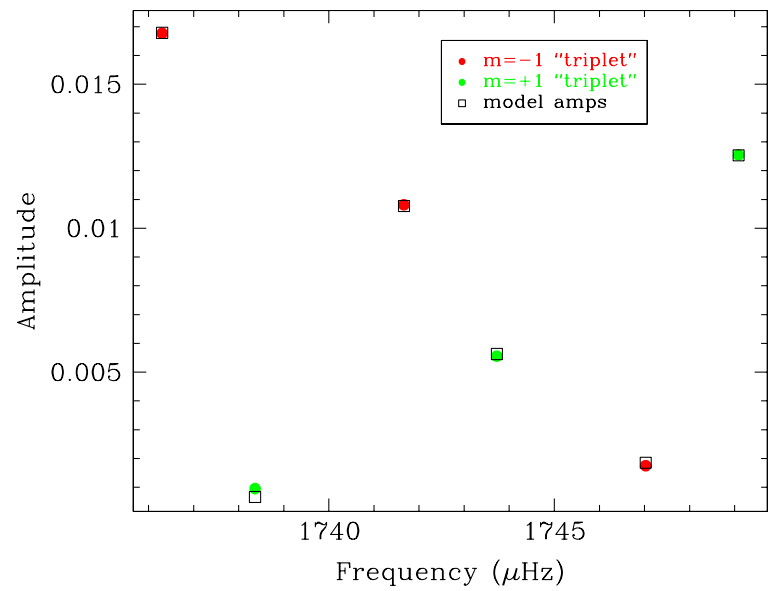

Figure 9. Fit of the calculated amplitudes (open squares) to the observed amplitudes (points) for the different components of " $k=12$ " assuming the oblique pulsator model. The errors on the observed amplitudes are smaller than the size of the points.

(A color version of this figure is available in the online journal.)

reason, the $m=0$ member of the original triplet is not present at observable amplitudes.

Finally, we wish to test the ability of the oblique pulsator model to adequately reproduce the amplitudes of the geometric peaks. To calculate the amplitudes we use the analytical expressions given in the Appendix (see Unno et al. 1989; Kurtz \& Shibahashi 1986), and to perform the fits we have used a genetic algorithm (Charbonneau 1995). This allows us to search the $m$ values of each intrinsic triplet as well as the values of the parameters $\theta_{i}, \beta$, and $x_{1}$. As we demonstrate below, such a fit is more constrained than one would think given the six data points and five free parameters.

In Figure 9, we show the result of the fit to the amplitudes in Table 4 . The fit is quite impressive. It has the added bonus that triplets 1 and 2 are required to originate from $|m|=1$ intrinsic modes and they must have opposite signs. This corroborates our

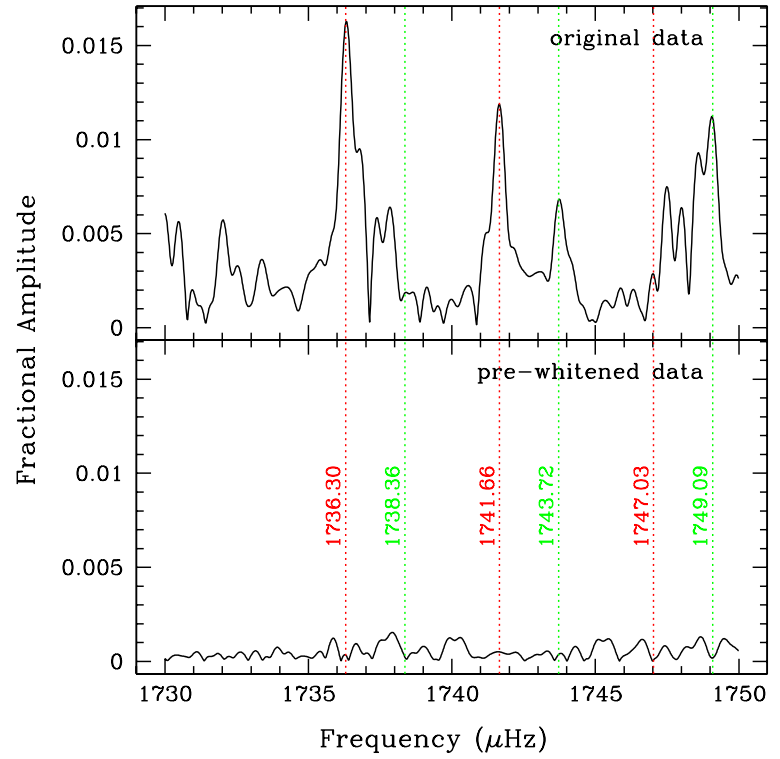

Figure 10. Pre-whitening of the same region as in Figure 8, but using the full oblique pulsator model for the amplitudes, frequencies, and phases. Thus, not only are the frequency splittings constrained, but the amplitudes within a triplet and their relative phases are as well.

(A color version of this figure is available in the online journal.)

earlier result based on the phases that both triplets originated from $|m|=1$ intrinsic modes.

Given the near equality of the number of data points and free parameters we wished to assess how easily our model could reproduce any data set. To do this, we randomly generated amplitudes for 500 pairs of triplets and fit them in the same way we fit the data, normalizing the residuals by the meansquared amplitudes of the two triplets. We found that in only 11 cases out of 500 were the random amplitudes better fit by our model than the measured amplitudes were. Thus, we conclude that our amplitude fits are significant at the $\sim 98 \%$ level.

As a final check on this procedure, we use these amplitudes together with Equations (A2)-(A4) to pre-whiten the $k=12$ region of the FT, as shown in Figure 10. Although considerably more constrained, this pre-whitening is clearly just as effective as that shown in Figure 8. Thus, the oblique pulsator model passes all tests with flying colors when applied to the $k=12$ region of the FT.

\subsection{Interpretation of the Oblique Pulsator Fits}

The relative amplitudes of the peaks within a triplet split by oblique pulsation/rotation depend on $\theta_{i}, \beta$, and $x_{1}$ (see Section 6.1 for a definition of these quantities). From the amplitude fits obtained in the previous section, we find that $\theta_{i}=46.3, \beta=31.8$, and $x_{1}=5.65$. This value of $\theta_{i}$ is close to that obtained from our nonlinear light curve fits, $47.5 \pm 2.2$ (Section 3). This provides an important consistency check on both methods.

Given that $\theta_{i}$ is the angle between the rotation axis and our line of sight and that $\beta$ is angle between the rotation and pulsation/magnetic axes, the effective inclination angle of a mode aligned with the magnetic axis should vary between $\theta_{i}-\beta$ and $\theta_{i}+\beta$ as the star rotates, i.e., between 14.5 and 78.1 . From the top panel of Figure 4, we see that $\theta_{i}$ varies with about half of this amplitude. The straightforward explanation for this is that only the $k=12$ modes are affected by oblique rotation, while the other modes are aligned with the rotation axis. As a 
result, the value of $\theta_{i}$ shown in Figure 4 represents the average inclination angle for all modes. Averaging over all modes yields an inclination angle which varies by about $15^{\circ}$ rather than the full $32^{\circ}$ experienced by the $k=12$ modes.

Finally, the parameter $x_{1}$ is a measure of the relative strength of the Coriolis and magnetic perturbations; the fit value $x_{1}=$ 5.65 indicates that the magnetic field is the dominant perturber. This reinforces the idea that the pulsations of these modes are tied to the magnetic axis. In addition, for a large-scale dipole field simple perturbation theory applied to $\ell=1$ and 2 modes yields

$$
\omega_{|m|}^{(1)} \propto\left\{\begin{array}{ll}
\frac{2}{5}+\frac{2}{5} m^{2}, & \ell=1 \\
\frac{18}{7}-\frac{2}{7} m^{2}, & \ell=2
\end{array},\right.
$$

where $\omega_{|m|}^{(1)}$ is the perturbation to the frequency of the mode due to the magnetic field (Montgomery 1994; Jones et al. 1989). Together with Equation (10), the fact that $x_{1}$ is positive means that $\omega_{0}^{(1)}>\omega_{1}^{(1)}$, which in turn suggests that $\ell \geqslant 2$. In reality, this indicates that these modes, at least in the surface layers, may not be pure $\ell=1$ modes but contain a mixture of other higher $\ell$ components.

The success of the oblique pulsator model in explaining the $k=12$ peaks constitutes evidence that oblique pulsation plays an important role in GD 358. However, there is little conclusive evidence that other modes in this star are undergoing oblique pulsation. On the contrary, the stable $k=8$ and 9 triplets are consistent only with the traditional case of the pulsation axis aligned with the rotation axis. A possible explanation is that the outer turning points for the $k=8$ and 9 modes are farther from the surface than they are for the $k=12$ modes, so if the dominant magnetic effects are also confined to the surface layers then the lower $k$-modes would not be affected by the magnetic field and the higher $k$-modes would.

The magnitudes of the multiplet splittings/fine structure also present a challenge. For instance, the oblique pulsator model requires a rotation frequency of $5.36 \mu \mathrm{Hz}$, whereas the splittings of the $k=8$ and 9 modes, believed to result from standard rotational splitting, give a value of $7.5 \mu \mathrm{Hz}$ (Provencal et al. 2009; Winget et al. 1994). Since different modes sample different regions of the star's interior, differential rotation in the radial direction could explain these results.

A further concern is the internal consistency of the oblique pulsator fit. The central components of each of the triplets in Table 4 should themselves be members of an intrinsic triplet which is split by rotation. This splitting is observed to be $2.06 \mu \mathrm{Hz}$ and should equal $2 C_{k \ell} f_{\text {rot }} \cos \beta$ for solid body rotation of the star. This is satisfied if $C_{k \ell} \sim 0.225$ instead of $C_{k \ell} \sim 0.5$ as is expected for $\ell=1$ modes. The splitting within a geometric triplet is a direct measure of the surface rotation rate, while the difference in frequency of the central components of these triplets (the "intrinsic" modes) is given by $2 C_{k \ell} f_{\text {rot }} \cos \beta$, where $f_{\text {rot }}$ represents a radial average of the rotation profile. Thus, differential rotation, with the interior rotating more slowly than the surface layers, could explain these values. An alternate explanation is that the magnetic field mixes higher $\ell$ components into the spatial structure of the $k=12$ modes. They would therefore have rotational constants $C_{k \ell}$ indicative of higher $\ell$-modes. Since $C_{k \ell} \sim 1 / \ell(\ell+1)$ for moderate to high- $k$ modes, these modes would have correspondingly smaller values of $C_{k \ell}$.

A further possibility is that the intrinsic modes have $\ell=2$ instead of $\ell=1$. In this case only three of the five possible geometric peaks would be large enough to be detectable. First, we note that the predicted phase relations are the same as for the $\ell=1$ case, so the measured phase relations support either case equally. Also, since $C_{k \ell} \sim 1 / 6$ for $\ell=2$, using the rotational splitting of the $k=8$ and 9 modes yields a splitting of $2 C_{k \ell} f_{\text {rot }} \cos \beta \sim 2.2 \mu \mathrm{Hz}$, which is close to the measured value of $2.06 \mu \mathrm{Hz}$. In this case, though, the value of the inclination angle, $\theta_{i}=22$. 1 , does not agree with that from the light curve fits. Also, the $\ell=2$ fits are somewhat less constrained than the $\ell=1$ fits: fitting 500 random amplitudes in the same way as the data, $12 \%$ of the fits were better than the fit to the data, making the $\ell=2$ fit significant at the $88 \%$ level.

Even so, given the ability of the oblique pulsator model to describe (1) the power in the $k=12$ region, (2) the phase relations between the geometric triplets, and (3) the relative amplitudes within the triplets (to some extent), we believe that the abovementioned inconsistencies do not warrant rejection of the model but rather point us in the direction of future improvements. For instance, the amplitude calculations include magnetic effects in a fairly simple way; more sophisticated treatments may be necessary to adequately model the observations. Also, differential rotation in the radial direction may be able to resolve the seeming discrepancy of the $C_{k, \ell}$ values for the $\ell=1$ case.

\section{DISCUSSION}

In many respects GD 358 is a "simple" star to analyze. For instance, the spacing between the periods of its multiplets suggests that we are looking at successive radial overtone numbers of $\ell=1$ modes. This is based both on the fact that (1) given its distance, mass, and luminosity, only $\ell=1$ modes allow a consistent solution (Bradley \& Winget 1994) and (2) in many cases these multiplets are well-defined triplets (Winget et al. 1994). Furthermore, as a DBV it has no hydrogen layer, so fewer parameters are needed to model its structure. Indeed, it is the best-studied white dwarf variable and significant constraints have been placed on its interior structure (Metcalfe et al. 2000; Metcalfe 2003).

On the other hand, GD 358 is in many respects a complicated star to model. For instance, while the amplitudes of its modes can be fairly constant during a WET run, over timescales of a few months the amplitudes can change significantly. The most extreme case of amplitude change is the previously discussed sforzando in which dramatic amplitude changes occurred on a timescale of a day or less. We currently have no theory that adequately describes these changes.

What we do know is that two independent lines of evidence indicate that GD 358 was hotter during the sforzando event. The first is that the average flux of GD 358 relative to the comparison stars is larger, and the second is that the nonlinear light curve fits indicate a thinner convection zone-presumably the result of an increase in surface temperature. If we assume that GD 358 remained hotter for approximately a day then such a temperature increase would require a total energy input of approximately $6 \times 10^{36} \mathrm{erg}$. While detailed nonadiabatic models of GD 358's pulsational state just prior to the sforzando would be required, rough estimates indicate that this amount of energy may typically be present in the higher $k$-modes. Thus, if these modes were somehow damped and deposited their pulsation energy in the surface layers of the star over the period of a day, this would explain GD 358's temporary temperature increase. Such a scenario would explain both the temperature increase and the disappearance of the high- $k$ modes, although more detailed models would be necessary to check quantitatively the energetics. 
We note that an increase in the apparent amplitude of the $k=8$ mode is a generic feature of a thinner convection zone (e.g., smaller value of $\tau_{0}$ ), although the predicted factor $(\sim 8)$ is still less than what was observed $(\sim 30)$. While speculative, it is also possible that some mechanism may have transferred power from the high- $k$ to the low- $k$ modes, although we currently do not understand how this would proceed. Perhaps least likely is the possibility that the hotter "equilibrium" state of the sforzando allowed mode growth and damping to occur on timescales given by linear theory and that these timescales were many orders of magnitude shorter than expected.

Another possibility is that the event was tied to a magnetic phenomenon and that this phenomenon somehow led to a temperature increase as well as a change in the amplitudes of the various modes. There are several reasons that this is not completely far-fetched. First, the oblique pulsator model used here requires a mechanism to align modes with an axis other than the rotation axis, and a magnetic field can provide this. Second, the $k=8$ and 9 triplets are asymmetric in the sense that the $m=0$ mode is closer to either the $m=+1$ or $m=-1$ mode (see Figures 18 and 19 of Provencal et al. 2009). Perhaps not coincidentally, the asymmetry seen in the splittings of the $k=8$ and 9 modes reversed themselves at the time of the sforzando, and this change has persisted ever since. This timescale of years is consistent with what is seen in the magnetic cycle of the Sun and other stars (Elsworth et al. 1990; Libbrecht \& Woodard 1990). Finally, small but definite shifts in frequency of order $0.5 \mu \mathrm{Hz}$ have been seen on timescales as short as 3 weeks. These could be a sign of magnetic activity: changes in the surface magnetic field could produce slight perturbations in the mode frequencies (Jones et al. 1989), and the timescale of a few weeks for these to take place again seems plausible.

A final unresolved issue for GD 358 is differential rotation. Taken at face value, the difference in triplet splitting between high $-k$ and low $-k$ modes implies significant differential rotation (Winget et al. 1994). In addition, the oblique pulsator model as applied in this paper implies differential rotation: the $k=12$ region requires a surface rotation rate of $\sim 2.17$ days whereas the $k=8$ and 9 splittings give a rotation rate of $\sim 1.5$ days. The $k=8$ and 9 splittings reflect a bulk average of the rotation rate whereas the frequency differences within the geometric triplets in the $k=12$ region give us the rate at the surface. In addition, the frequency splitting between the intrinsic modes in the $k=12$ region also implies differential rotation, albeit with the interior rotating less rapidly than the surface. Previously, detailed examinations of the frequency splittings in GD 358 as a function of $k$ have been inconclusive (Kawaler et al. 1999), but such analyses did not take into account the possibility of oblique pulsation.

An intriguing possibility is that the pulsations themselves lead to differential rotation. Townsend (2009) has recently shown that $g$-modes in massive stars can transport angular momentum relatively rapidly compared to evolutionary timescales. This transport occurs predominantly in regions in which the mode is driven and/or damped and leads to differential rotation. If this effect occurs in white dwarfs then it could alter the rotation rate from the surface down past the base of the convection zone into the radiative damping layers. If the rotation profile of these outer layers is continually changing then this would also explain the small shifts in frequency which have been detected in GD 358. In addition, a more dramatic shift in the rotation profile could be associated with the sforzando event. Perhaps the outer part of GD 358's rotation profile experienced a shift during this event and it has persisted in its new state for the past several years. This would explain the shift in asymmetry of the $k=8$ and 9 triplets that also occurred at this time (see Provencal et al. 2009).

\section{CONCLUSIONS}

In this paper, we have extended our nonlinear light curve fitting technique to the multiperiodic pulsator GD 358. Our fit to the 2006 WET data provides a good match to the light curves and we find that the thermal response time of its convection zone is $\tau_{0}=572.9 \pm 6.1 \mathrm{~s}$. This is considerably larger than that of the star PG1351+489, for which $\tau_{0} \sim 100 \mathrm{~s}$ (Montgomery 2005). This difference in $\tau_{0}$ is consistent with the effective temperatures of these stars: the pure He solution for PG1351+489 yields a $T_{\text {eff }}$ which is $\sim 2000 \mathrm{~K}$ hotter than that for GD 358 .

We also obtained a fit to the light curve of GD 358 during the sforzando event in 1996. These fits showed that GD 358 had $\tau_{0} \sim 42 \pm 2 \mathrm{~s}$, a value much less than that determined from the 2006 data. This suggests that its effective temperature was approximately $2000 \mathrm{~K}$ hotter in 1996 than in 2006, and this is consistent with the estimate of Weidner \& Koester (2003) that the light curve shape suggests $T_{\text {eff }} \sim 27,000 \mathrm{~K}$. Independent evidence of GD 358's brightness relative to comparison stars is also consistent with such a temperature increase at the time of the sforzando (Provencal et al. 2009). The physical origin of this temperature increase will be the subject of future work.

As expected, these data indicate an increase in the depth/ mass of the convection zone with decreasing $T_{\text {eff. }}$ A similar trend is given by ML2 $/ \alpha=1.1$ convection (Böhm \& Cassinelli 1971), although the slope of the theoretical relation appears less steep than that of the data. In addition, lower values of $\alpha \lesssim 0.6$ are excluded. In general, $\tau_{0}$ is also a function of $\log g$, albeit a somewhat weaker one. Our ultimate goal is to map $\tau_{0}$ as a function of both $T_{\text {eff }}$ and $\log g$ for both the DBV and DAV instability strips. These data will provide insight into the physics of convection, still one of the largest uncertainties in stellar modeling. They will also serve as important constraints for new hydrodynamic simulations of convection which are starting to come online.

Multiple lines of evidence point to some of GD 358's modes undergoing oblique pulsation, in particular, the peaks in the $k=12$ region. First, these peaks can be fit with two sets of exactly evenly spaced triplets. Second, the relative phases of each of the components within the triplet indicate that each originates as a single $m=-1$ or +1 mode aligned with the magnetic axis; as the star rotates, the magnetic axis precesses around the rotation axis, generating a triplet for each intrinsic mode. Finally, the oblique pulsator model qualitatively and quantitatively fits the amplitudes of the peaks seen in the FT. Taken together, this marks the first time that oblique pulsation has been seen in a white dwarf variable.

Having now identified the characteristics of oblique pulsation in GD 358 we now know what to look for in other white dwarf variables; we have found preliminary indications of it in other stars and in other data sets of GD 358. As discussed in the previous sections, oblique pulsation may prove to be a diagnostic of both the magnetic field and its changes as well as a diagnostic of differential rotation. This opens an exciting chapter in the seismology of these objects.

This research was supported in part by the Delaware Asteroseismic Research Center, the National Science Foundation 
under grant AST-0909107, and the Norman Hackerman Advanced Research Program under grant 003658-0255-2007. A.J.S. thanks the National Science Foundation for support under grant AST-0607840. The Delaware Asteroseismic Research
Center (DARC) is grateful for the support of the Crystal Trust Foundation and Mt. Cuba Observatory. DARC also acknowledges the support of the University of Delaware, through their participation in the SMARTS consortium.

\section{APPENDIX}

\section{EQUATIONS OF OBLIQUE PULSATION FOR $\ell=1$ AND 2 MODES}

The formulae given below are taken from Unno et al. (1989). Following Kurtz \& Shibahashi (1986), we use $\omega$ instead of $\sigma$ for the angular frequencies of the modes. We use $\omega^{(0)}$ for the unperturbed frequency of the mode in a non-rotating, non-magnetic star, and $\omega_{|m|}^{(1)}$ for the perturbation to this frequency due to the magnetic field (but not due to rotation). The pulsation axis is assumed to be aligned with the magnetic axis, which makes an angle of $\beta$ with the rotation axis, and $\theta_{i}$ is taken to be the angle between the rotation axis and our line of sight. As the pulsation and magnetic axes rotate around the star, we find that a mode of given frequency and $\{\ell, m\}$ values is split into a triplet of peaks. We tabulate below the resulting time dependence of modes having the given values of $\ell$ and $m$. In the following, $x_{1}$ is defined to be

$$
x_{1} \equiv \frac{\omega_{0}^{(1)}-\omega_{1}^{(1)}}{C \Omega},
$$

where $C$ is the rotational splitting coefficient due to the Coriolis force for $\ell=1$ modes and $\Omega$ is the angular velocity of rotation at the stellar surface. Since $x_{1}$ is the ratio of the rotational splitting to the splitting induced by the magnetic field it provides a useful estimate of the relative importance of the two effects. Also, since it depends on the difference in magnetic splitting between $m=0$ and $|m|=1$ modes, it is sensitive to the geometry of the magnetic field. With these definitions, the luminosity perturbations associated with $\ell=1$ oblique pulsation are given below:

$\ell=1, m=-1$ :

$$
\begin{aligned}
\Delta L / L= & \frac{1}{\sqrt{2}}\left(1-\frac{1+\cos \beta}{x_{1}}\right) \sin ^{2} \frac{\beta}{2} \sin \theta_{i} \cos \left[\left(\omega^{(0)}+\omega_{1}^{(1)}-C \Omega \cos \beta-\Omega\right) t+\phi\right] \\
& +\frac{1}{\sqrt{2}}\left(1-\frac{\cos \beta}{x_{1}}\right) \sin \beta \cos \theta_{i} \cos \left[\left(\omega^{(0)}+\omega_{1}^{(1)}-C \Omega \cos \beta\right) t+\phi\right] \\
& -\frac{1}{\sqrt{2}}\left(1+\frac{1-\cos \beta}{x_{1}}\right) \cos ^{2} \frac{\beta}{2} \sin \theta_{i} \cos \left[\left(\omega^{(0)}+\omega_{1}^{(1)}-C \Omega \cos \beta+\Omega\right) t+\phi\right]
\end{aligned}
$$

$\ell=1, m=0:$

$$
\begin{aligned}
\Delta L / L= & \frac{1}{2}\left(1+\frac{1}{x_{1}}\right) \sin \beta \sin \theta_{i} \cos \left[\left(\omega^{(0)}+\omega_{0}^{(1)}-\Omega\right) t+\phi\right] \\
& +\cos \beta \cos \theta_{i} \cos \left[\left(\omega^{(0)}+\omega_{0}^{(1)}\right) t+\phi\right] \\
& +\frac{1}{2}\left(1-\frac{1}{x_{1}}\right) \sin \beta \sin \theta_{i} \cos \left[\left(\omega^{(0)}+\omega_{0}^{(1)}+\Omega\right) t+\phi\right]
\end{aligned}
$$

$\ell=1, m=1$ :

$$
\begin{aligned}
\Delta L / L= & \frac{1}{\sqrt{2}}\left(1-\frac{1-\cos \beta}{x_{1}}\right) \cos ^{2} \frac{\beta}{2} \sin \theta_{i} \cos \left[\left(\omega^{(0)}+\omega_{1}^{(1)}+C \Omega \cos \beta-\Omega\right) t+\phi\right] \\
& -\frac{1}{\sqrt{2}}\left(1+\frac{\cos \beta}{x_{1}}\right) \sin \beta \cos \theta_{i} \cos \left[\left(\omega^{(0)}+\omega_{1}^{(1)}+C \Omega \cos \beta\right) t+\phi\right] \\
& -\frac{1}{\sqrt{2}}\left(1+\frac{1+\cos \beta}{x_{1}}\right) \sin ^{2} \frac{\beta}{2} \sin \theta_{i} \cos \left[\left(\omega^{(0)}+\omega_{1}^{(1)}+C \Omega \cos \beta+\Omega\right) t+\phi\right]
\end{aligned}
$$

More generally for arbitrary $\ell$ and $m$, we define $x_{|m|}$

$$
x_{|m|} \equiv \frac{\omega_{0}^{(1)}-\omega_{|m|}^{(1)}}{C \Omega}
$$

where $C$ is the rotational splitting coefficient for the appropriate $\ell$ values. With these definitions, the luminosity perturbations associated with $\ell=2$ oblique pulsation are given below:

$\ell=2, m=-2$ :

$$
\Delta L / L=\sqrt{\frac{3}{8}} \sin ^{4} \frac{\beta}{2} \sin ^{2} \theta_{i}\left(1+\frac{2(1+\cos \beta)}{x_{1}-x_{2}}\right) \cos \left[\left(\omega^{(0)}+\omega_{2}^{(1)}-2 C \Omega \cos \beta-2 \Omega\right) t+\phi\right]
$$


No. 1, 2010

$$
\begin{aligned}
& +\sqrt{\frac{3}{8}} \sin ^{2} \frac{\beta}{2} \sin \beta \sin 2 \theta_{i}\left(1+\frac{1+2 \cos \beta}{x_{1}-x_{2}}\right) \cos \left[\left(\omega^{(0)}+\omega_{2}^{(1)}-2 C \Omega \cos \beta-\Omega\right) t+\phi\right] \\
& +\frac{1}{8} \sqrt{\frac{3}{2}} \sin ^{2} \beta\left(1+3 \cos 2 \theta_{i}\right)\left(1+\frac{2 \cos \beta}{x_{1}-x_{2}}\right) \cos \left[\left(\omega^{(0)}+\omega_{2}^{(1)}-2 C \Omega \cos \beta\right) t+\phi\right] \\
& -\sqrt{\frac{3}{8}} \cos ^{2} \frac{\beta}{2} \sin \beta \sin 2 \theta_{i}\left(1+\frac{-1+2 \cos \beta}{x_{1}-x_{2}}\right) \cos \left[\left(\omega^{(0)}+\omega_{2}^{(1)}-2 C \Omega \cos \beta+\Omega\right) t+\phi\right] \\
& +\sqrt{\frac{3}{8}} \cos ^{4} \frac{\beta}{2} \sin ^{2} \theta_{i}\left(1+\frac{2(-1+\cos \beta)}{x_{1}-x_{2}}\right) \cos \left[\left(\omega^{(0)}+\omega_{2}^{(1)}-2 C \Omega \cos \beta+2 \Omega\right) t+\phi\right]
\end{aligned}
$$

$\ell=2, m=-1$ :

$$
\begin{aligned}
\Delta L / L & =\frac{1}{8} \sqrt{\frac{3}{2}} \sin \beta \sin ^{2} \theta_{i}\left(2-2 \cos \beta-\frac{3 \sin ^{2} \beta}{x_{1}}-\frac{(1-\cos \beta)^{2}}{x_{1}-x_{2}}\right) \cos \left[\left(\omega^{(0)}+\omega_{1}^{(1)}-C \Omega \cos \beta-2 \Omega\right) t+\phi\right] \\
& +\frac{1}{4} \sqrt{\frac{3}{2}} \sin 2 \theta_{i}\left[1+\cos \beta-2 \cos ^{2} \beta+\sin ^{2} \beta\left(-\frac{3 \cos \beta}{x_{1}}+\frac{-1+\cos \beta}{x_{1}-x_{2}}\right)\right] \cos \left[\left(\omega^{(0)}+\omega_{1}^{(1)}-C \Omega \cos \beta-\Omega\right) t+\phi\right] \\
& +\frac{1}{16} \sqrt{\frac{3}{2}} \sin \beta\left(1+3 \cos 2 \theta_{i}\right)\left[4 \cos \beta-\frac{1+3 \cos 2 \beta}{x_{1}}-\frac{2 \sin ^{2} \beta}{x_{1}-x_{2}}\right] \cos \left[\left(\omega^{(0)}+\omega_{1}^{(1)}-C \Omega \cos \beta\right) t+\phi\right] \\
& -\frac{1}{4} \sqrt{\frac{3}{2}} \sin 2 \theta_{i}\left[-1+\cos \beta+2 \cos ^{2} \beta+\sin ^{2} \beta\left(\frac{3 \cos \beta}{x_{1}}-\frac{1+\cos \beta}{x_{1}-x_{2}}\right)\right] \cos \left[\left(\omega^{(0)}+\omega_{1}^{(1)}-C \Omega \cos \beta+\Omega\right) t+\phi\right] \\
& -\frac{1}{8} \sqrt{\frac{3}{2}} \sin \beta \sin ^{2} \theta_{i}\left(2+2 \cos \beta+\frac{3 \sin ^{2} \beta}{x_{1}}+\frac{(1-\cos \beta)^{2}}{x_{1}-x_{2}}\right) \cos \left[\left(\omega^{(0)}+\omega_{1}^{(1)}-C \Omega \cos \beta+2 \Omega\right) t+\phi\right]
\end{aligned}
$$

$\ell=2, m=0$ :

$$
\begin{aligned}
\Delta L / L= & \frac{3}{8} \sin ^{2} \beta \sin ^{2} \theta_{i}\left(1+\frac{2}{x_{1}}\right) \cos \left[\left(\omega^{(0)}+\omega_{0}^{(1)}-2 \Omega\right) t+\phi\right] \\
& +\frac{3}{8} \sin 2 \beta \sin 2 \theta_{i}\left(1+\frac{1}{x_{1}}\right) \cos \left[\left(\omega^{(0)}+\omega_{0}^{(1)}-\Omega\right) t+\phi\right] \\
& +\frac{1}{16}(1+3 \cos 2 \beta)\left(1+3 \cos 2 \theta_{i}\right) \cos \left[\left(\omega^{(0)}+\omega_{0}^{(1)}\right) t+\phi\right] \\
& +\frac{3}{8} \sin 2 \beta \sin 2 \theta_{i}\left(1-\frac{1}{x_{1}}\right) \cos \left[\left(\omega^{(0)}+\omega_{0}^{(1)}+\Omega\right) t+\phi\right] \\
& +\frac{3}{8} \sin ^{2} \beta \sin ^{2} \theta_{i}\left(1-\frac{2}{x_{1}}\right) \cos \left[\left(\omega^{(0)}+\omega_{0}^{(1)}+2 \Omega\right) t+\phi\right]
\end{aligned}
$$

$\ell=2, m=+1:$

$$
\begin{aligned}
\Delta L / L & =\frac{1}{8} \sqrt{\frac{3}{2}} \sin \beta \sin ^{2} \theta_{i}\left(2+2 \cos \beta-\frac{3 \sin ^{2} \beta}{x_{1}}-\frac{(1+\cos \beta)^{2}}{x_{1}-x_{2}}\right) \cos \left[\left(\omega^{(0)}+\omega_{1}^{(1)}-C \Omega \cos \beta-2 \Omega\right) t+\phi\right] \\
& +\frac{1}{4} \sqrt{\frac{3}{2}} \sin 2 \theta_{i}\left[-1+\cos \beta+2 \cos ^{2} \beta+\sin ^{2} \beta\left(-\frac{3 \cos \beta}{x_{1}}+\frac{1+\cos \beta}{x_{1}-x_{2}}\right)\right] \cos \left[\left(\omega^{(0)}+\omega_{1}^{(1)}-C \Omega \cos \beta-\Omega\right) t+\phi\right] \\
& -\frac{1}{16} \sqrt{\frac{3}{2}} \sin \beta\left(1+3 \cos 2 \theta_{i}\right)\left[4 \cos \beta+\frac{1+3 \cos 2 \beta}{x_{1}}+\frac{2 \sin ^{2} \beta}{x_{1}-x_{2}}\right] \cos \left[\left(\omega^{(0)}+\omega_{1}^{(1)}-C \Omega \cos \beta\right) t+\phi\right] \\
& -\frac{1}{4} \sqrt{\frac{3}{2}} \sin 2 \theta_{i}\left[1+\cos \beta-2 \cos ^{2} \beta+\sin ^{2} \beta\left(\frac{3 \cos \beta}{x_{1}}+\frac{1-\cos \beta}{x_{1}-x_{2}}\right)\right] \cos \left[\left(\omega^{(0)}+\omega_{1}^{(1)}-C \Omega \cos \beta+\Omega\right) t+\phi\right] \\
& +\frac{1}{8} \sqrt{\frac{3}{2}} \sin \beta \sin ^{2} \theta_{i}\left(-2+2 \cos \beta-\frac{3 \sin ^{2} \beta}{x_{1}}-\frac{(1-\cos \beta)^{2}}{x_{1}-x_{2}}\right) \cos \left[\left(\omega^{(0)}+\omega_{1}^{(1)}-C \Omega \cos \beta+2 \Omega\right) t+\phi\right]
\end{aligned}
$$

$\ell=2, m=+2$ :

$$
\begin{aligned}
\Delta L / L & =\sqrt{\frac{3}{8}} \cos ^{4} \frac{\beta}{2} \sin ^{2} \theta_{i}\left(1+\frac{2(1-\cos \beta)}{x_{1}-x_{2}}\right) \cos \left[\left(\omega^{(0)}+\omega_{2}^{(1)}-2 C \Omega \cos \beta-2 \Omega\right) t+\phi\right] \\
& -\sqrt{\frac{3}{8}} \cos ^{2} \frac{\beta}{2} \sin \beta \sin 2 \theta_{i}\left(1+\frac{1-2 \cos \beta}{x_{1}-x_{2}}\right) \cos \left[\left(\omega^{(0)}+\omega_{2}^{(1)}-2 C \Omega \cos \beta-\Omega\right) t+\phi\right]
\end{aligned}
$$




$$
\begin{aligned}
& +\frac{1}{8} \sqrt{\frac{3}{2}} \sin ^{2} \beta\left(1+3 \cos 2 \theta_{i}\right)\left(1-\frac{2 \cos \beta}{x_{1}-x_{2}}\right) \cos \left[\left(\omega^{(0)}+\omega_{2}^{(1)}-2 C \Omega \cos \beta\right) t+\phi\right] \\
& +\sqrt{\frac{3}{8}} \sin ^{2} \frac{\beta}{2} \sin \beta \sin 2 \theta_{i}\left(1-\frac{1+2 \cos \beta}{x_{1}-x_{2}}\right) \cos \left[\left(\omega^{(0)}+\omega_{2}^{(1)}-2 C \Omega \cos \beta+\Omega\right) t+\phi\right] \\
& +\sqrt{\frac{3}{8}} \sin ^{4} \frac{\beta}{2} \sin ^{2} \theta_{i}\left(1-\frac{2(1+\cos \beta)}{x_{1}-x_{2}}\right) \cos \left[\left(\omega^{(0)}+\omega_{2}^{(1)}-2 C \Omega \cos \beta+2 \Omega\right) t+\phi\right]
\end{aligned}
$$

\section{REFERENCES}

Beauchamp, A., Wesemael, F., Bergeron, P., Fontaine, G., Saffer, R. A., Liebert, J., \& Brassard, P. 1999, ApJ, 516, 887

Bertelli, G., Nasi, E., Girardi, L., \& Marigo, P. 2009, A\&A, 508, 355

Böhm, K. H., \& Cassinelli, J. 1971, A\&A, 12, 21

Bradley, P. A., \& Winget, D. E. 1994, ApJ, 430, 850

Brickhill, A. J. 1983, MNRAS, 204, 537

Brickhill, A. J. 1991, MNRAS, 251, 673

Brickhill, A. J. 1992, MNRAS, 259, 519

Castanheira, B. G., Nitta, A., Kepler, S. O., Winget, D. E., \& Koester, D. 2005, A\&A, 432, 175

Chandrasekhar, S. 1939, An Introduction to the Study of Stellar Structure (Chicago: Univ. Chicago Press)

Charbonneau, P. 1995, ApJS, 101, 309

Di Mauro, M. P., Christensen-Dalsgaard, J., Kjeldsen, H., Bedding, T. R., \& Paternò, L. 2003, A\&A, 404, 341

Elsworth, Y., Howe, R., Isaak, G. R., McLeod, C. P., \& New, R. 1990, Nature, 345,322

Fontaine, G., \& Brassard, P. 2008, PASP, 120, 1043

Goldreich, P., \& Wu, Y. 1999, ApJ, 511, 904

Ising, J., \& Koester, D. 2001, A\&A, 374, 116

Jones, P. W., Hansen, C. J., Pesnell, W. D., \& Kawaler, S. D. 1989, ApJ, 336, 403

Kawaler, S. D., Sekii, T., \& Gough, D. 1999, ApJ, 516, 349

Kepler, S. O., et al. 2003, A\&A, 401, 639

Koester, D. 2010, Mem. Soc. Astron. Ital., in press (arXiv:0812.0482)
Kurtz, D. W. 1982, MNRAS, 200, 807

Kurtz, D. W., \& Shibahashi, H. 1986, MNRAS, 223, 557

Libbrecht, K. G., \& Woodard, M. F. 1990, Nature, 345, 779

Mestel, L. 1952, MNRAS, 112, 583

Metcalfe, T. S. 2003, ApJ, 587, L43

Metcalfe, T. S., Nather, R. E., \& Winget, D. E. 2000, ApJ, 545, 974

Montgomery, M. H. 1994, Masters thesis, Univ. Texas, Austin

Montgomery, M. H. 2005, ApJ, 633, 1142

Montgomery, M. H. 2007, in ASP Conf. Ser. 372, 15th European Workshop on White Dwarfs, ed. A. Napiwotzki \& M. R. Burleigh (San Francisco, CA: ASP), 635

Montgomery, M. H. 2008, Commun. Asteroseismol., 154, 38

Muthsam, H. J., Kupka, F., Löw-Baselli, B., Obertscheider, C., Langer, M., \& Lenz, P. 2010, New Astron., 15, 460

Provencal, J. L., et al. 2009, ApJ, 693, 564

Robinson, E. L., Kepler, S. O., \& Nather, R. E. 1982, ApJ, 259, 219

Townsend, R. 2009, in AIP Conf. Proc. 1170, Stellar Pulsation: Challenges for Theory and Observation, ed. J. A. Guzik \& P. A. Bradley (Melville, NY: AIP), 355

Unno, W., Osaki, Y., Ando, H., Saio, H., \& Shibahashi, H. 1989, Nonradial Oscillations of Stars (Tokyo: Univ. Tokyo Press)

Watson, R. D. 1988, Ap\&SS, 140, 255

Weidner, C., \& Koester, D. 2003, A\&A, 406, 657

Winget, D. E., \& Kepler, S. O. 2008, ARA\&A, 46, 157

Winget, D. E., et al. 1994, ApJ, 430, 839

Wu, Y. 2001, MNRAS, 323, 248

Wu, Y., \& Goldreich, P. 1999, ApJ, 519, 783 